\title{
A Face Serum Containing Palmitoyl Tripeptide-38, Hydrolyzed Hyaluronic Acid, Bakuchiol and a Polyherbal and Vitamin Blend Improves Skin Quality
}

\author{
Brett J. West ${ }^{1}$, Ifedayo Alabi ${ }^{2}$, Shixin Deng1 \\ ${ }^{1}$ Research and Development Department, NewAge, Inc., American Fork, Utah, USA \\ ${ }^{2}$ Research and Development Department, NewAge, Inc., Bray, Republic of Ireland \\ Email: ^brett_west@newage.com
}

How to cite this paper: West, B.J., Alabi, I. and Deng, S.X. (2021) A Face Serum Containing Palmitoyl Tripeptide-38, Hydrolyzed Hyaluronic Acid, Bakuchiol and a Polyherbal and Vitamin Blend Improves Skin Quality. Journal of Cosmetics, Dermatological Sciences and Applications, 11, 237-252.

https://doi.org/10.4236/jcdsa.2021.113020

Received: July 29, 2021

Accepted: September 15, 2021

Published: September 18, 2021

Copyright $\odot 2021$ by author(s) and Scientific Research Publishing Inc. This work is licensed under the Creative Commons Attribution International License (CC BY 4.0).

http://creativecommons.org/licenses/by/4.0/

\begin{abstract}
A face serum composed of a combination of biologically active compounds was evaluated for safety and efficacy in vitro, in a repeat insult patch test and in a human clinical efficacy trial. The serum inhibited tyrosinase activity modestly, decreased collagenase activity and exhibited notable free radical scavenging activity in vitro. It is gentle to the skin, as the serum did not irritate the skin or produce symptoms of allergic contact dermatitis in the 55 healthy adults that participated in the repeat insult patch test. In the efficacy trial, daily application of the face serum for 30 days significantly increased skin hydration, with all 35 volunteers experiencing improvement. Substantial improvements in skin elasticity, roughness (fine lines and wrinkles), and brightness also occurred during the trial. Dermatological examination also revealed a trend for reduced comedone count with use of the serum. Selfassessment responses revealed that all volunteers experienced improvements in multiple skin quality parameters and that participant perceptions are consistent with the results of the instrumental analyses. These findings indicated that the measured improvements in skin quality are not only statistically significant but are also clinically relevant as they were great enough for users of the face serum to feel and recognize.
\end{abstract}

\section{Keywords}

Face Serum, Bakuchiol, Palmitoyl Tripeptide-38, Hyaluronic Acid, Repeat Insult Patch Test, Tyrosinase, Collagenase, Antioxidant, Clinical Trial 


\section{Introduction}

The skin is a major protective organ of the human body. It provides first-line protection against numerous potential threats in our environment. The skin also helps regulate the temperature of our bodies, is the site of vitamin D synthesis and can produce certain hormones [1]. The skin is an important means of social communication, and the status of skin health can have a significant psychosocial impact individually as well as affect social interactions [2] [3] [4] [5]. Further, skin quality has become a major indicator of social worth in many modern societies [6]. As such, efforts to maintain skin health will benefit physical and mental health as well interpersonal relationships.

Many extrinsic and intrinsic factors can accelerate the appearance of aging skin. Excessive UV light exposure, environment, lifestyle habits and genetic predisposition all influence the quality of human skin. A decrease in skin radiance, development of uneven melanin distribution and appearance of rhytides and telangiectasia are examples of visible signs of underlying degradation of extracellular matrix during the aging process. Topical skin treatments may potentially slow these skin changes and, perhaps, even restore the skin to a previous state of quality. Among the substances that may promote skin health are plant-derived compounds. One notable example is bakuchiol, a meroterpene which occurs in Psoralea corylifolia. Bakuchiol exhibits antioxidant and anti-inflammatory activities as well as possesses retinol-like antiaging properties but without the associated adverse effects [7] [8].

Terminalia ferdinandiana (Kakadu plum) is a source of several antioxidant compounds including vitamin $\mathrm{C}$ which, when topically applied, protects against UV-induced photo aging by possibly increasing gene expression of the tissue inhibitor of matrix metalloproteinases [9] [10]. Shea butter, which is the phytosterol and tocopherol-rich fat extracted from the nut of the Vitellaria paradoxa tree, has long been used in sub-Saharan Africa as a folk remedy for dermatological disorders and has also found wide acceptance as an emollient which helps maintain skin barrier integrity [11] [12]. Perhaps, Aloe vera (syn. Aloe barbadensis) is one of the best-known natural products for skin health. Topical Aloe vera gel may aid burn and wound healing as well as act as an anti-inflammatory agent [13]. Allantoin, derived from several plant sources, regulates inflammation and induces both fibroblast proliferation and extracellular matrix synthesis [14]. Case reports indicate that topical application of grape seed oil reduced the appearance of scaring and dark circles under the eyes [15].

Peptides have also demonstrated antiaging potential. Due to their role in cell signaling within the skin, peptides have been investigated in the past few decades for topical benefits. Specific peptides have been developed to cross the stratum corneum and influence extracellular matrix synthesis, pigmentation, immunity and inflammation [16]. Palmitoyl tripeptide-38 is one such lipopeptide which is reported to increase the production of dermal and epidermal collagen, fibronectin and hyaluronic acid [17].

Vitamin C (ascorbic acid) improves skin elasticity and reduces wrinkles by 
stimulating collagen synthesis. It inhibits tyrosinase, which is responsible for the formation of melanin. It also protects the skin from oxidative stress by donating electrons to reactive oxygen species (ROS) neutralizing their effect. However, ascorbic acid is an extremely unstable compound. Ascorbyl phosphate salts, such as sodium ascorbyl phosphate (SAP), are among one of the most stable ascorbic acid derivatives. This is due to the introduction of the phosphate group that protects the enediol system of the molecule against oxidation. It is converted to free vitamin C by enzymes present in the skin. The liposomal form of SAP not only further enhances stability but also exhibits greater penetration through the epidermis, thereby exerting greater effect on skin cells [18]

Hyaluronic acid is a component of the skin and integral to skin function via its ability to retain water. As the skin ages, epidermal hyaluronic acid content dramatically declines. The disappearance of this water-binding molecule results in the characteristic age-associated decrease in skin hydration status [19]. Unsurprisingly, topical application of sodium hyaluronate and other forms of hyaluronic acid have been found to effectively improve skin hydration, resulting in improvements in various facets of skin quality [20].

The purpose of our investigation was to evaluate both the safety and efficacy of a face serum developed with the substances discussed above. The serum was formulated to provide a comprehensive antiaging topical treatment which would mitigate the adverse effects of environmental and intrinsic factors on the extracellular matrix as well as reduce some of the visible effects of aging. Such a formulation could aid users in their efforts to maintain a youthful appearance and promote overall well-being.

\section{Materials and Methods}

\subsection{Test Materials}

A face serum (Lucim) was formulated and prepared for daily application to the face. The serum was composed of an aqueous base with the following percentages of International Nomenclature of Cosmetic Ingredients (INCI) categories (with active ingredients in descending order of predominance): $9.7005 \%$ skin conditioning agents (grape seed oil, niacinamide, shea butter, allantoin, Aloe barbadensis leaf juice, sodium hyaluronate and palmitoyl tripeptide-38), 5.3795\% humectants (glycerin, sorbitol and hydrolyzed hyaluronic acid), 2.0025\% emollients (sucrose stearate, coconut alkanes, coco-caprylate/caprate and glyceryl caprylate) and $1.3 \%$ antioxidants (bakuchiol, sodium ascorbyl phosphate, tocopherol and Kakadu plum fruit extract). The $\mathrm{pH}$ of the serum was 5. It was prepared in accordance with internationally recognized good manufacturing practices. To ensure product safety, microbiological analyses were performed on samples of the serum following production and an antimicrobial effectiveness test was conducted in accordance with harmonized US and EU protocols.

\subsection{Repeat Insult Patch Test}

Fifty-five volunteers (13 males and 42 females, age 18 to 79 years) were enrolled 
in a repeat insult patch test of the face serum. The inclusion criteria for the repeat insult patch test were healthy adult volunteers with no physical or skin conditions which would interfere with the ability to discern potential skin reactions. During the induction phase, $0.2 \mathrm{~mL}$ of the face serum was placed on cotton fabric patch and applied for 24 hours to the skin of the back, with semi occlusive surgical tape, adjacent to the spinal and between the scapulae and the waist. For each volunteer, application occurred 3 times a week with 24-hour rest periods between each application, except for a 48-hour rest period between the final weekly application and the first application of the following week. The procedure was followed for 3 weeks, resulting in 9 applications during the induction phase. Two weeks after completion of the induction phase, a patch containing the face serum was applied to a previously unexposed section of skin. The skin of each subject was evaluated during this challenge phase at 24 and 72 hours following the final application of the face serum for possible delayed reactions, including redness, itching, and irritation. The skin of the volunteers was scored for reactions according to the International Contact Dermatitis Research Group and under the supervision of a consulting dermatologist [21]. Reactions were scored on a scale of 0 (no reaction) to 4 (deep red erythema, with or without vesiculation or weeping).

\subsection{Tyrosinase Inhibition Assay}

In vitro tyrosinase inhibition was evaluated according to a previously described method, but with modifications [22]. Various concentrations of sample were dissolved in $180 \mu \mathrm{L}$ phosphate buffer $\left(193 \mathrm{mM} \mathrm{KH}_{2} \mathrm{PO}_{4}, 73.4 \mathrm{mM} \mathrm{Na}_{2} \mathrm{CO}_{3}, \mathrm{pH}\right.$ 7.2) with $0.5 \mathrm{mM} \mathrm{L-dopa} \mathrm{(Sigma-Aldrich,} \mathrm{St.} \mathrm{Louis,} \mathrm{Missouri)} \mathrm{and} 1400$ units tyrosinase (Sigma-Aldrich, St. Louis, Missouri) and incubated at room temperature. A $100 \%$ activity blank (no sample) was also prepared. These mixtures were incubated at room temperature for 15 minutes. Afterwards, absorbance was read at $450 \mathrm{~nm}$ in a microplate reader. Replicate samples were evaluated at each concentration. Tyrosinase inhibition activity was calculated from difference between $100 \%$ activity (no sample) and sample absorbance, divided by the absorbance of the $100 \%$ activity alone.

\subsection{Collagenase Inhibition Assay}

Inhibition of collagenase activity was assessed using an assay kit obtained from Sigma-Aldrich. In this assay, samples were prepared at various concentrations with deionized water and filtered via $0.45 \mu \mathrm{M}$ syringe filter. The dissolved samples $(2 \mu \mathrm{L})$ or a solvent blank (initial enzyme activity) were combined with $10 \mu \mathrm{L}$ collagenase solution $(0.35$ units $/ \mathrm{mL})$ and $88 \mu \mathrm{L}$ buffer in a clear polystyrene microwells then incubated at room temperature for 10 minutes. Next, an equal volume $(100 \mu \mathrm{L})$ of assay buffer, containing the collagenase substrate $\mathrm{N}$-(3-[2Furyl]acryloyl)-Leu-Gly-Pro-Ala (FALGPA), was added to each well containing samples or blank. The absorbance of each reaction mixture was measured at 345 $\mathrm{nm}$ in kinetic mode (every minute for 15 minutes) while incubating at $37^{\circ} \mathrm{C}$. The 
area under the curve (AUC) for the absorbance vs. time of each sample or blank was calculated. AUC values were used to calculate inhibition, which was the difference between values for the blank and each sample, divided by that of the blank alone. Blanks and samples were evaluated in triplicate.

\subsection{DPPH Radical Scavenging Assay}

The 2,2-Diphenylpicrylhydrazyl (DPPH) radical scavenging assay was performed according to a method reported previously, but with some modification [23]. In the DPPH test, samples of increasing concentrations were prepared with aqueous ethanol. Next, sample solutions were combined 1:1 (v/v) with $0.4 \mathrm{mM}$ DPPH in ethanol. The absorbance of each sample and blank (solvent only) was read at $515 \mathrm{~nm}$ after incubation at $37^{\circ} \mathrm{C}$ for $1 \mathrm{hr}$. Percent radical scavenging activity was calculated by dividing the difference in blank absorbance and sample by the absorbance of the blank alone. In the in vitro assays, sample concentrations are reported on dry weight basis (water weight excluded).

\subsection{Efficacy Clinical Trial}

A one-month clinical trial involving 30 healthy volunteers was conducted with the face serum. All participants agreed to not use other topical products on the face 7 days prior to the start of the study as well as during remainder of the study period. Volunteers excluded from the trial were pregnant or lactating women, those who had cosmetic facial treatments within the previous 4 weeks, those undergoing immunosuppressive therapy, current use of topical medications, allergy or hypersensitivity to one of the face serum ingredients, or a medical history of atopy, keloids, or postinflammatory hyperpigmentation. The demographics of those enrolled in the trial were age range of 35 to 55 years, 23 females of European ancestry, 2 females of both European and African ancestry, 4 females of Asian ancestry, and 1 male of Asian ancestry. The percentage of facial skin types among the participants were $15 \%$ dry, $22 \%$ normal, $63 \%$ combination and none with oily skin.

The face serum was applied to the face once daily for 4 weeks. Visual evaluation by a dermatologist was performance before the start of the trial (T0), and after 4 weeks (T2) of facial application of the face serum. Clinical scores were given to initial and final conditions of the facial skin (forehead, cheeks, cheekbone, nose and chin) of each volunteer regarding the number of comedones and inflammatory lesions.

Instrumental analysis of the skin was performed at $\mathrm{T} 0$, two weeks after daily use of the face serum (T1) and at T2. Skin elasticity was measured with the $\mathrm{Cu}$ tometer dual MPA 580 (Courage + Khazaka, Cologne, Germany) using mode 1, with a probe (model CT580MP) aperture of $2 \mathrm{~mm}$ and 450 mbar negative pressure (suction). Total skin recovery at the end of deformation cycles (Ua), immediate skin retraction (Ur), and final skin deformation (Uf), all measured in millimeters, were used to calculate skin elasticity $R$ values. Biological elasticity (R2) is the ratio Ua/Uf, while relative elastic recovery (R7) is the ratio Ur/Uf [24]. 
Skin hydration was measured with a Corneometer ${ }^{\oplus}$ CM 825 probe (Cour-age + Khazaka). This is one of the most well-documented noninvasive methods for reproducibly and accurately measuring the moisture level of the stratum corneum [25]. This method is based on capacitance measurements of the skin, in which the dielectric constant will change with water content. The probe is applied to the surface of the skin at a constant pressure of the measurement probe on the skin surface is 3.5 newtons, covers an area of $49 \mathrm{~mm}^{2}$, and measures water content up to a depth of $100 \mu \mathrm{m}$. Skin hydration measurements were conducted in a clinic room maintained at $22^{\circ} \mathrm{C} \pm 2{ }^{\circ} \mathrm{C}$ and $50 \%$ relative humidity, the same conditions under which the other measurements were made. Skin hydration measurements were reported in corneometer units (CU).

Facial skin roughness (lateral canthal lines or wrinkles) was measured with DermaTOP Blue (Eotech SA, Marcoussis, France), a blue LED device for noncontact 3D analysis of skin roughness via fringe projection and optical triangulation. This allows for the measurement of skin wrinkles without the need to produce silicone skin replicates. The roughness $(\mathrm{R})$ parameters $\mathrm{Rz}$ and $\mathrm{Ra}$, both measured in $\mathrm{mm}$, were evaluated. $\mathrm{Rz}$ is defined as the average wrinkle height/ depth. $\mathrm{Ra}$ is the average wrinkle roughness, calculated from wrinkle width and height [26].

Skin brightness (specular brightness rate) was measured with a FrameScan ${ }^{\circledR}$ (ORION Concept, Tours, France). Changes in reflected light, which is relative to skin brightness, were measured with this system and used to calculate specular brightness rates. All instrument measurements performed under the same conditions as those use for skin hydration measurements.

At the end of the trial participants were asked to complete a self-assessment evaluation wherein they were instructed to look in a mirror and complete a questionnaire on noticeable differences in the appearance of their facial skin face and on their perception of efficacy and pleasantness face serum.

Summary statistics, such as mean and standard deviation, were calculated for each of the measurements. Data were evaluated for normality with the Shapiro-Wilk test followed by the paired Student's t-test for differences among measurements taken at $\mathrm{T} 0, \mathrm{~T} 1$ and $\mathrm{T} 2$. Where data was not normally distributed, the Wilcoxon signed-rank test was used to make comparisons. The percent change in each measurement for individual participants was also determined and used to calculate average $\%$ change. Both the efficacy trial and the repeat insult patch test were conducted in accordance with the World Medical Association's Declaration of Helsinki, and written informed consent was obtained from all participants.

\section{Results}

\subsection{Repeat Insult Patch Test}

All 55 subjects completed the repeat insult patch test. There were no reactions observed during the induction phase. Further, there were no clinically significant 
responses during the challenge phase. As such, the face serum is not associated with skin irritation or allergic contact dermatitis.

\subsection{Tyrosinase Inhibition Assay}

In vitro tests of the face serum reveal at least a few potentially effective mechanisms of action. Anti-tyrosinase activity was found to be concentration-dependent but very modest. Tyrosinase was inhibited as low as $25 \mu \mathrm{g} / \mathrm{mL}$. However, no more than $20 \%$ inhibition was achieved at a concentration of $1 \mathrm{mg} / \mathrm{mL}$.

\subsection{Collagenase Inhibition Assay}

The face serum was more active against collagenase, demonstrating linear and concentration-dependent inhibition (Figure 1). Inhibition of collagenase was still evident at $3 \mu \mathrm{g} / \mathrm{mL}$, and half-maximal inhibition (IC50) was achieved by 300 $\mu \mathrm{g} / \mathrm{mL}$

\subsection{DPPH Radical Scavenging Assay}

The face serum was much more active in the DPPH radical scavenging assay (Figure 2). It reduced free radicals in a concentration dependent manner, where the average ( \pm standard deviation) activity was $92.72 \% \pm 0.0006 \%$ at $150 \mu \mathrm{g} / \mathrm{mL}$. Even at a concentration as low as $10 \mu \mathrm{g} / \mathrm{mL}$, the actives in the face serum were able to neutralize approximately $20 \%$ of the free radicals in this assay.

\subsection{Efficacy Clinical Trial}

The average ( \pm standard deviation) of parameters measured during the trial are presented in Table 1. Over the course of the efficacy trial, all participants experienced increases in skin hydration. After 2 weeks of face serum use, average skin moisture among the volunteers increased $9.28 \%$, from (mean \pm standard deviation) $41.853 \pm 12.391$ to $45.740 \pm 11.356 \mathrm{CU}, \mathrm{p}<0.001$. By the end of the trial, average skin moisture increased $23.82 \%$ from baseline (up to $50.520 \pm$ $11.823, \mathrm{p}<0.001)$. The averages of the percent change experienced by individual participants during the first and second halves of the trial were approximately equal (11.59\% and $23.82 \%$, respectively), demonstrating a consistent response to the moisturizing properties of the face serum.

Skin elasticity increased in $97 \%$ of participants after 30 days. Mean biological elasticity (R2) increased $10 \%$ after 2 weeks of face serum use (from $0.556 \pm 0.060$ to $0.609 \pm 0.062, \mathrm{p}<0.001)$. Average R2 increased further to $0.641 \pm 0.054$ ( $\mathrm{p}<$ 0.001 ) after 30 days, where the average of individual percent increase was $15 \%$ above baseline (T0). The mean elastic recovery ability of the skin (R7) improved by approximately $8 \%$ within the first two weeks of the trial (from $0.382 \pm 0.055$ to $0.414 \pm 0.067, \mathrm{p}<0.01)$. As with $\mathrm{R} 2$ values, average $\mathrm{R} 7$ continued to improve until the end of the trial where it increased by about $13 \%$ (up to $0.430 \pm 0.047, \mathrm{p}$ $<0.01$ ). Improvements in R2 and R7 demonstrate improvements in both elastin and collagen fibers of the skin as well as moisture content, likely via increased skin hyaluronic acid content. 


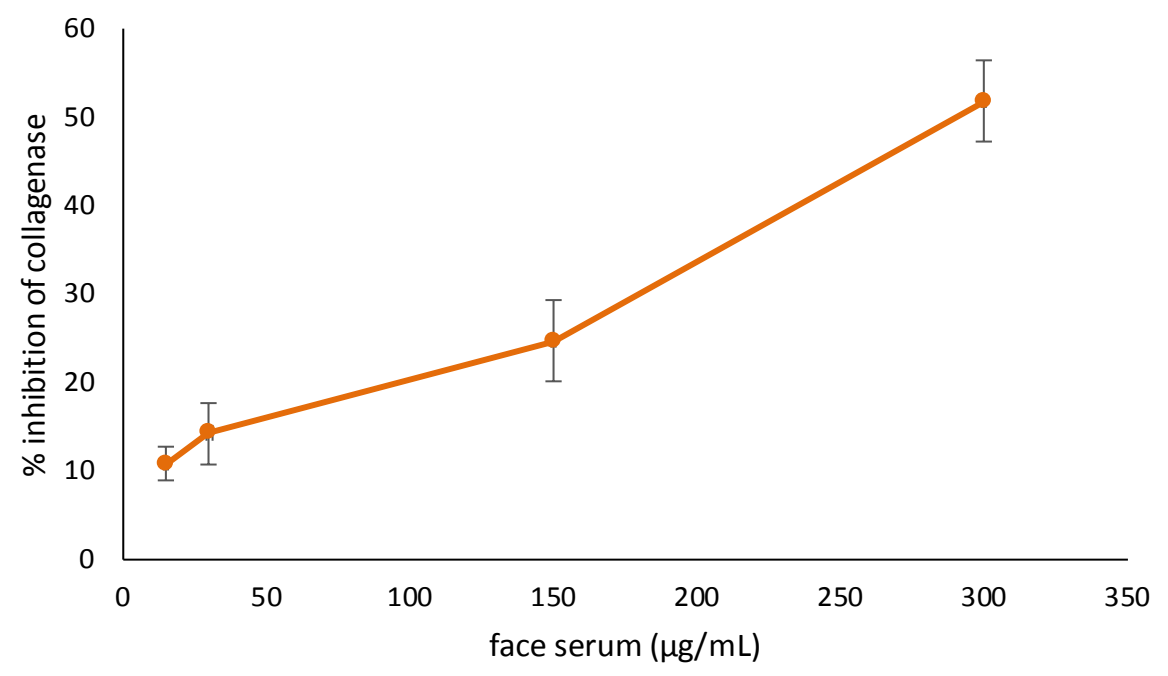

Figure 1. Inhibition of collagenase by face serum.

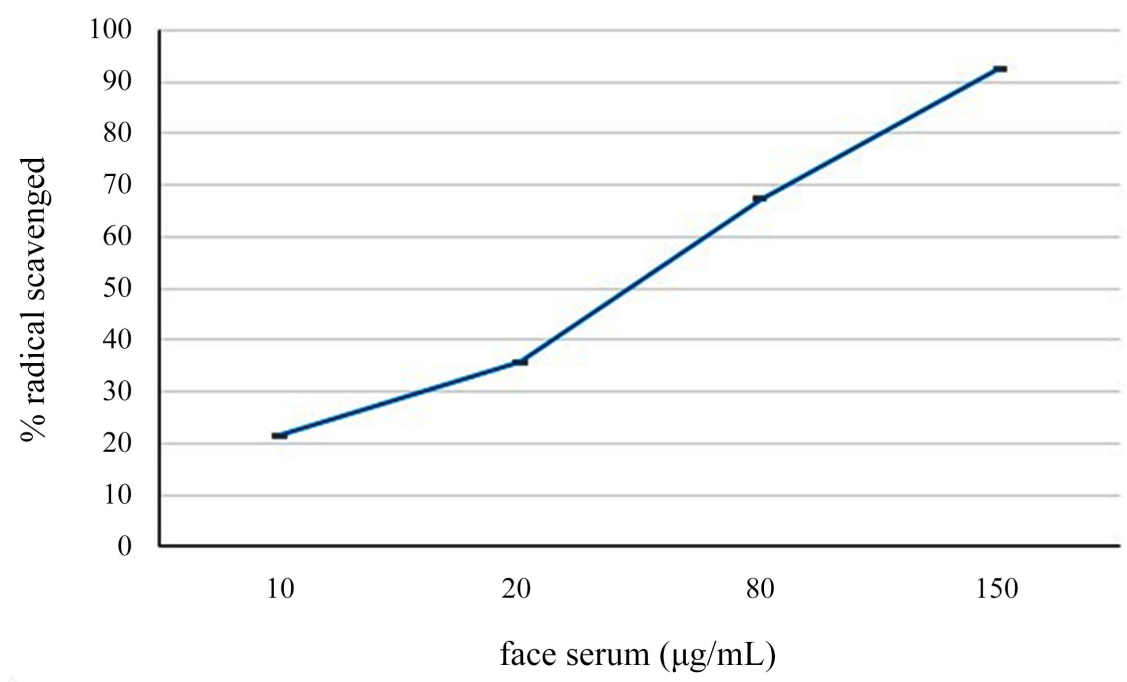

Figure 2. DPPH radical scavenging test of face serum.

Table 1. Average ( \pm standard deviation) of measured parameters at the start (T0) of the efficacy trial as well as 15 days (T1) and 30 days (T2) after daily application of face serum.

\begin{tabular}{cccc}
\hline Parameter & T0 & T1 & T2 \\
\hline Moisture (CU) & $41.853 \pm 12.391$ & $45.740 \pm 11.356^{*}$ & $50.520 \pm 11.823^{*}$ \\
Biological elasticity (R2) & $0.556 \pm 0.060$ & $0.609 \pm 0.062^{\star}$ & $0.641 \pm 0.054^{*}$ \\
Relative elastic recovery (R7) & $0.382 \pm 0.055$ & $0.414 \pm 0.067^{*}$ & $0.430 \pm 0.047^{*}$ \\
Average wrinkle roughness, Ra (mm) & $0.044 \pm 0.012$ & $0.042 \pm 0.012^{*}$ & $0.038 \pm 0.012^{*}$ \\
Average wrinkle height, Rz (mm) & $0.163 \pm 0.046$ & $0.155 \pm 0.045^{*}$ & $0.143 \pm 0.043^{*}$ \\
Specular brightness rate & $0.322 \pm 0.220$ & $0.365 \pm 0.211^{*}$ & $0.403 \pm 0.216^{*}$ \\
\hline
\end{tabular}

${ }^{*} \mathrm{p}<0.01$ vs. T0.

Skin wrinkle width and depth parameters ( $\mathrm{Ra}$ and $\mathrm{Rz}$, both measured in $\mathrm{mm}$ ) decreased by an average of $5.5 \%$ and $4.9 \%$ after 2 weeks ( $\mathrm{p}<0.01)$ and by $12.5 \%$ 
and $12.2 \%$ after 1 month ( $\mathrm{p}<0.01$ ), respectively. All participants, except one, experienced a decrease of in both $\mathrm{Ra}$ and $\mathrm{Rz}$ skin roughness values after 30 days of treatment. An example of the decrease in lateral canthal lines in given in Figure 3. The subject in these images experienced an $11.5 \%$ decline in $\mathrm{Rz}$ values over the course of the trial as well as an $8.10 \%$ decrease in Ra values. The greater decrease in $\mathrm{Rz}$ values is readily discernable with the reduction in the amount of violet color, which corresponds to the greatest wrinkle depth, in the images captured during the latter two measurement dates. The participant featured in the images in Figure 3 had experience somewhat modest results when compared to 14 other volunteers who experience greater reductions in $\mathrm{Rz}$ values. Larger declines in $\mathrm{Ra}$ values that of the person in Figure 3 were also observed in twenty participants. The most substantial declines in $\mathrm{Rz}$ and $\mathrm{Ra}$ values were both $29.10 \%$.

The average brightness rate values rose from $0.322 \pm 0.220$ at T0 to $0.403 \pm$ 0.216 at $\mathrm{T} 2(\mathrm{p}<0.01)$. But the average individual percent change in specular brightness rate, from $\mathrm{T} 0$ to $\mathrm{T} 1$, was $22.5 \%$. By the end of the trial, this increased to $37.8 \%$. As with other parameters measured in this trial, all participants experienced an increase in specular brightness of the skin. The most dramatic change occurred in a Caucasian female whose values nearly doubled from 0.113 to 0.224 , a $98.23 \%$ increase. However, there was no evidence of any ancestry/ethnicity-dependent differential in skin brightness improvement, as there
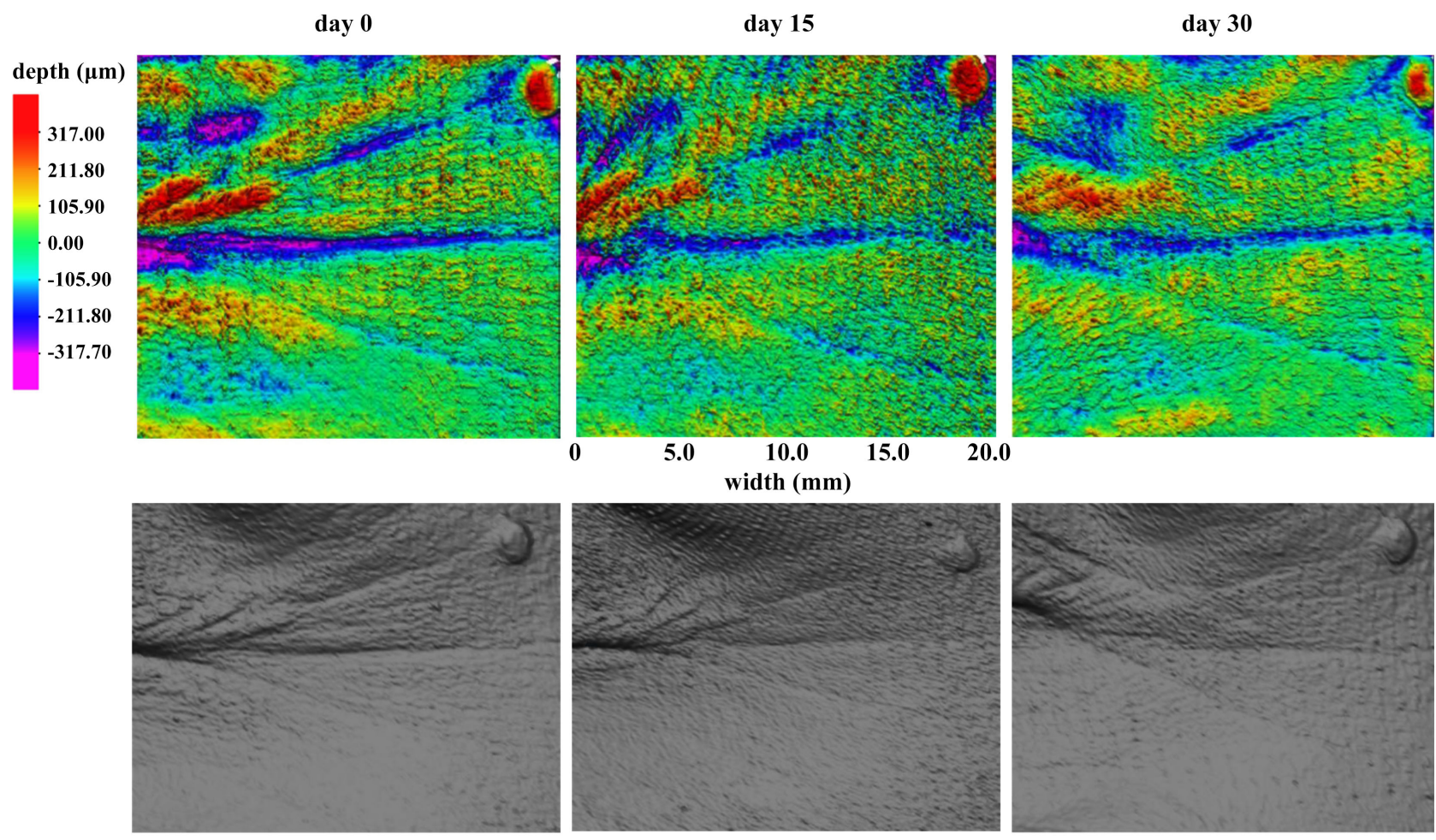

Figure 3. Example case of change in skin roughness (wrinkle depth and width) of lateral canthal lines, as visualized with DermaTOP Blue analysis. Corresponding black and white photos of the same facial area are given immediately below the depth and width color-scaled photos. 
were overlapping minimum-maximum ranges for each skin type- $4.4 \%$ to $25.3 \%$ for those of both African and European ancestry, $0.9 \%$ to $51.3 \%$ for those of Asian ancestry and $2.7 \%$ to $98.23 \%$ for those of European only ancestry. It can be reasonably expected that if a larger number of participants of Asian and African ancestry were included in the trial, even greater responses would be observed. It should also be noted that more than two-thirds of the trial participants experience greater than $10 \%$ increase in skin brightness, with a little over one-third having increases of $50 \%$ or more.

Dermatological evaluation of the facial skin of the trial participants revealed that the face serum did not increase the occurrence of seborrhea or increase the number of large pores. While not statistically significant, there was a trend of decreased comedones after one month of face serum application, especially on the cheek and forehead (Table 2).

In the self-assessment (Table 3), all participants reported experiencing improved in skin hydration. For the question regarding whether participants felt their skin was hydrated, the number reporting "not at all" or "a little" decreased from a combined $41 \%$ to $0 \%$ by the end of the trial, while the percent reporting "a lot" increased from $0 \%$ to $15 \%$ and those reporting "enough" increased by $26 \%$. This perception is consistent with the instrumental measurements. Most participants also reported experiencing significant increases in skin brightness, softness, radiance, elasticity, tone, and smoothness. All participants reported an overall improvement in facial skin, reduction in wrinkles and improvement in skin vitality after 1 month of face serum use. Among all self-assessment questions, no participants responded with "a lot" at T0. However, a substantial number of participants responded with "a lot" to the self-assessment questions by the end of the trial. Most volunteers noticed significant improvements in the crow's feet area. As with improved in skin hydration, these perceptions are consistent with instrumental analyses and subsequent statistical analyses. This indicates the measured changes in skin quality are substantial enough for users of the face serum to feel and recognize them.

Table 2. Number of comedones at start of trial (T0) and 30 days (T2) after daily application of face serum.

\begin{tabular}{ccccc}
\hline & \multicolumn{2}{c}{$\begin{array}{c}\text { Total number comedones } \\
\text { among participants }\end{array}$} & \multicolumn{2}{c}{$\begin{array}{c}\text { Number of comedones/person } \\
\text { (average } \pm \text { S.D.) }\end{array}$} \\
\cline { 2 - 5 } & T0 & T2 & T0 & T2 \\
\hline Forehead & 53 & 34 & $1.77 \pm 0.73$ & $1.13 \pm 0.43$ \\
Cheekbone & 33 & 31 & $1.10 \pm 0.31$ & $1.03 \pm 0.18$ \\
Nose & 57 & 40 & $1.90 \pm 0.48$ & $1.33 \pm 0.48$ \\
Cheeks & 41 & 31 & $1.37 \pm 0.56$ & $1.03 \pm 0.18$ \\
Chin & 55 & 38 & $1.83 \pm 0.53$ & $1.27 \pm 0.52$ \\
\hline
\end{tabular}


Table 3. Response percentage to self-assessment questions asked at the start of the efficacy trial (T0) and 30 days (T2) after daily application of face serum.

\begin{tabular}{|c|c|c|c|c|c|}
\hline & & \multicolumn{4}{|c|}{ Percentage of total responses } \\
\hline & & A lot & Enough & A little & Not at all \\
\hline \multirow{2}{*}{ How hydrated does your skin feel? } & T0 & 0 & 59 & 37 & 4 \\
\hline & $\mathrm{T} 2$ & 15 & 85 & 0 & 0 \\
\hline \multirow{2}{*}{ How bright is your skin? } & T0 & 0 & 37 & 41 & 22 \\
\hline & $\mathrm{T} 2$ & 7 & 81 & 11 & 0 \\
\hline \multirow{2}{*}{ How soft is your skin? } & T0 & 11 & 63 & 22 & 4 \\
\hline & T2 & 30 & 67 & 4 & 0 \\
\hline \multirow[b]{2}{*}{ How radiant is your skin? } & T0 & 0 & 37 & 41 & 22 \\
\hline & $\mathrm{T} 2$ & 26 & 59 & 15 & 0 \\
\hline \multirow{2}{*}{ How firm is your skin? } & T0 & 0 & 63 & 26 & 11 \\
\hline & $\mathrm{T} 2$ & 26 & 52 & 22 & 0 \\
\hline \multirow{2}{*}{ How elastic is your skin? } & T0 & 0 & 59 & 30 & 11 \\
\hline & $\mathrm{T} 2$ & 30 & 59 & 11 & 0 \\
\hline \multirow{2}{*}{ How smooth is your skin? } & T0 & 0 & 52 & 44 & 4 \\
\hline & $\mathrm{T} 2$ & 30 & 56 & 11 & 4 \\
\hline \multirow{2}{*}{ How tone is your skin? } & T0 & 0 & 44 & 48 & 7 \\
\hline & $\mathrm{T} 2$ & 15 & 74 & 7 & 4 \\
\hline \multicolumn{6}{|c|}{ Questions at conclusion of trial only } \\
\hline $\begin{array}{l}\text { How much has your facial } \\
\text { skin improved overall? }\end{array}$ & & 19 & 67 & 15 & 0 \\
\hline $\begin{array}{l}\text { How much more vital } \\
\text { does your skin look? }\end{array}$ & & 26 & 70 & 4 & 0 \\
\hline $\begin{array}{l}\text { Has the appearance of wrinkles } \\
\text { and/or fine lines improved? }\end{array}$ & & 19 & 59 & 22 & 0 \\
\hline $\begin{array}{l}\text { How satisfied are you with the } \\
\text { efficacy of the face serum? }\end{array}$ & & 40 & 56 & 4 & 0 \\
\hline
\end{tabular}

The collective results of the repeat insult patch test and the efficacy clinical trial demonstrate that daily use of the face serum can provide benefits to skin health. In the efficacy trial, improvements occurred in each participant. This was especially true for moisture content and specular brightness. Skin hydration influences interkeratinocyte reflectance as well as other skin quality factors [27]. So, it naturally follows that improved hydration of the stratum corneum and underlying tissue will result in the outcomes observed in this study. The in vitro tyrosinase assay results demonstrated that the face serum had inhibitory activity, even at low concentrations. However, the activity was only modest. Therefore, it appears likely that the improvements in skin brightness are more dependent on hydrating properties of the face serum than on anti-tyrosinase potential. 
In addition to the hydrating properties of the face serum, its ability to protect the extracellular matrix is evident in the results of the in vitro assay and clinical trial. The notable anti-collagenase activity will, of course, greatly impact the skin elasticity. Collagen degradation in the skin's extracellular matrix is a major aspect of the normal aging process. The resulting structural alterations reduce skin strength and resiliency, resulting in more prominent wrinkle formation [28]. Even so, the in vitro DPPH scavenging activity of the face serum points to significant antioxidant potential which may be the major factor in the positive outcomes observed in the efficacy trial.

With photoaging, collagen degradation and the associated adverse effects on skin quality are accelerated even further [29] [30]. Photoaging begins with UV-induced generation of reactive oxygen species (ROS), where skin cell chromophores are excited in the presence of molecular oxygen. This produces ROS such as superoxide anion radicals (SAR), hydroxyl radicals and hydrogen peroxide [31]. When excessive UV exposure overwhelms endogenous antioxidant systems, ROS initiate a cascade of cellular signals leading to the transcription of collagenases (matrix metalloproteinases, or MMPs) in fibroblasts and an influx of elastase producing neutrophils [32] [33]. Increased MMP and elastase activity is responsible for extracellular matrix changes that result in the appearance of wrinkling, sagging, and other structural changes in the skin. Therefore, the reduction in skin roughness and improvements in skin elasticity are not only the results of collagenase inhibition but are likely the result of potent antioxidant activity of the ingredients in the face serum, such as bakuchiol and Kakadu plum extract. Bakuchiol is an excellent scavenger of linoleic acid peroxyl radicals, the DPPH radical, hydroxyl radicals, and glutathiyl radicals [34]. The antioxidant activity of bakuchiol is also known to prevent elevated expression of metalloproteinase-9 induction in vivo [35]. Further, Kakadu plum inhibited the induction of matrix metallo-proteinase-1 gene expression and subsequent collagen degradation in the skin UV-B exposed mice [36].

Aside from collagenase inhibition and antioxidant action, which prevent degradation of the extracellular matrix, some ingredients in the face serum promote the synthesis of new extracellular components. As discussed previously, palmitoyl tripetide-38 application increases the production of dermal and epidermal collagen, fibronectin and hyaluronic acid. Formulations containing this tripeptide have been previously evaluated in clinical trials. However, their efficacies have varied due to differences in ingredient composition and the face serum evaluated in the current study has a greater demonstrable impact on skin health. For example, the average $\mathrm{Rz}$ value declined by $9 \%$, with improvements recorded for $70 \%$ of volunteers, in a 28 -day trial of a palmitoyl tirpetptide-38, vitamin C and vitamin E blend involving 35 women [37]. In our study, the face serum decreased average $\mathrm{Rz}$ values by $12.2 \%$, with recorded improvements for 29 out of 30 participants (97\%). Another trial evaluated the effect of daily application of a palmitoyl tripeptide-38, apple stem cell extract, creatine and urea blend on 32 
women. This specific blend had a measurable anti-wrinkle effect in $71 \%$ of the women. The average increase in skin hydration (by Corneometer) was $13 \%$, with an average increase in skin elasticity (by Cutometer) was 10\% after 28 days [38]. In our trial, the face serum improved wrinkles in $100 \%$ of the volunteers, improved average skin moisture by $23.82 \%$ and increase R2 and R7 elasticity measurements by $15 \%$ and $13 \%$, respectively. In a third clinical trial, 37 females evaluated the efficacy of a blend of palmitoyl tripeptide-38, rosemary leaf extract, tetrahexyldecylascorbate, Astragalus root extract and CoQ10 by self-assessment questionnaire after 12 weeks of daily topical application to the face [39]. After 4 weeks of use, only $21 \%$ of the subjects reported an improvement in the appearance of wrinkles. After 12 weeks, $83 \%$ reported improved skin hydration. But in our study, $100 \%$ of the subjects reported improvements in both wrinkles and hydration within just one month. The ability of several compounds in the face serum to increase the hyaluronic acid content of the skin certainly limits transepidermal water loss, thereby enhancing moisture. The improved response observed in our study suggests that the combination of palmitoyl tripeptide-38, bakuchiol, Kakadu plum extract, allantoin, etc. in our face serum is more effective in improving skin hydration and the extracellular matrix.

\section{Conclusion}

The face serum is gentle to the skin. The combination of biologically active compounds in the serum possesses collagenase inhibiting properties and notable antioxidant activity in vitro. Clinical trial results also reveal that this combination may have a greater impact on skin health than topical treatments which may contain some of the same ingredients. It seems that the skin hydrating properties of the face serum are responsible, to a major degree, for the improvements in skin brightness and viscoelasticity. Additionally, protection of the extracellular matrix by inhibition of ROS-induced damage and increased expression of genes controlling dermal collagen, elastin and hyaluronic acid synthesis appear to be likely contributors to the improvements in skin quality. The qualitative assessments, or perception, of the participants match the instrumental analyses and demonstrate that the face serum's impact on skin health is clinically relevant. Therefore, daily topical application of the combination of active compounds in the face serum is a convenient and effective means of protecting facial skin and reducing the all too common signs of aging.

\section{Acknowledgements}

The repeat insult patch test was completed with the assistance of John A. Erianne, Toni F. Miller and Annemarie E. Hollenback of Essex Testing Clinic, Inc. (Verona, New Jersey, USA). The efficacy trial was completed with the assistance of Massimo Gola and Chiara Chiaratti of Merieux NutriSciences Corporation (Prato, Italy). Lucim Totale Face Serum was provided by Ariix LLC (American Fork, Utah, USA). 


\section{Conflicts of Interest}

The authors declare no conflicts of interest regarding the publication of this paper.

\section{References}

[1] Zouboulis, C.C. (2000) Human Skin: An Independent Peripheral Endocrine Organ. Hormone Research in Paediatrics, 54, 230-242. https://doi.org/10.1159/000053265

[2] Morrison, I., Loken, L.S. and Olausson, H. (2010) The Skin as a Social Organ. Experimental Brain Research, 204, 305-314. https://doi.org/10.1007/s00221-009-2007-y

[3] Jafferany, M. (2007) Psychodermatology: A Guide to Understanding Common Psychocutaneous Disorders. Primary Care Companion to the Journal of Clinical Psychiatry, 9, 203-213. https://doi.org/10.4088/PCC.v09n0306

[4] Tuckman, A. (2017) The Potential Psychological Impact of Skin Conditions. Dermatology and Therapy, 7, 53-57. https://doi.org/10.1007/s13555-016-0169-7

[5] Nichols, T. (2018) Health Utility, Social Interactivity, and Peristomal Skin Status: A Cross Sectional Study. Journal of Wound, Ostomy and Continence Nursing, 45, 438-443. https://doi.org/10.1097/WON.0000000000000457

[6] Honigman, R. and Castle, D.J. (2006) Aging and Cosmetic Enhancement. Clinical Interventions in Aging, 1, 115-119.

[7] Dhaliwal, S., Rybak, I., Ellis, S.R., Notay, M., M Trivedi, M., Burney, W., Vaughn, A.R., Nguyen, M., Reiter, P., Bosanac, S., Yan, H., Foolad, N. and Sivamani, R.K. (2019) Prospective, Randomized, Double-Blind Assessment of Topical Bakuchiol and Retinol for Facial Photoageing. British Journal of Dermatology, 180, 289-296. https://doi.org/10.1111/bjd.16918

[8] Chaudhuri, R.K. and Bojanowski, K. (2014) Bakuchiol: A Retinol-Like Functional Compound Revealed by Gene Expression Profiling and Clinically Proven to Have Anti-Aging Effects. International Journal of Cosmetic Science, 36, 221-230. https://doi.org/10.1111/ics.12117

[9] Mohanty, S. and Cock, I.E. (2012) The Chemotherapeutic Potential of Terminalia ferdinandiana: Phytochemistry and Bioactivity. Pharmacognosy Reviews, 6, 29-36. https://doi.org/10.4103/0973-7847.95855

[10] Al-Niaimi, F. and Chiang, N.Y.Z. (2017) Topical Vitamin C and the Skin: Mechanisms of Action and Clinical Application. Journal of Clinical and Aesthetic Dermatology, 10, 14-17.

[11] Ugwu-Dike, P. and Nambudiri, V.E. (2021) A Review of the Ethnomedicinal Uses of Shea Butter for Dermatoses in Sub-Saharan Africa. Dermatologic Therapy, e14786. (Online Ahead of Print) https://doi.org/10.1111/dth.14786

[12] Andersson, A.-C. and Alander, J. (2015) Shea Butter Extract for Bioactive Skin Care. Cosmetics and Toiletries, 130, 18-25.

[13] Feily, A. and Namazi, M.R. (2009) Aloe Vera in Dermatology: A Brief Review. Giornale Italiano di Dermatologia e Venereologia, 144, 85-91.

[14] Araujo, L. U., Grabe-Guimaraes, A., Mosqueira, V.C.F., Carneiro, C.M. and Silva-Barcellos, N.M. (2010) Profile of Wound Healing Process Induced by Allantoin. Acta Cirúrgica Brasileira, 25, 460-461. https://doi.org/10.1590/S0102-86502010000500014

[15] Spiers, S.M. and Cleaves, F.T. (1999) Topical Treatment of the Skin with a Grape- 
seed Oil Composition. US Patent No. 5916573A.

[16] Schagen, S.K. (2017) Topical Peptide Treatments with Effective Anti-Aging Results. Cosmetics, 4, Article No. 16. https://doi.org/10.3390/cosmetics4020016

[17] Ferreira, S.M., Magalhaes, M.C., Sousa-Lobo, J.M. and Almeida, I.F. (2020) Trending Anti-Aging Peptides. Cosmetics, 7, Article No. 91. https://doi.org/10.3390/cosmetics7040091

[18] Foco, A., Gasperlin, M. and Kristl, J. (2005) Investigation of Liposomes as Carriers of Sodium Ascorbyl Phosphate for Cutaneous Photoprotection. International Journal of Pharmaceutics, 291, 21-29. https://doi.org/10.1016/j.ijpharm.2004.07.039

[19] Papakonstantinou, E., Roth, M. and Karakiulakis, G. (2012) Hyaluronic acid: a key molecule in skin aging. Dermato-Endocrinology, 4, 253-258. https://doi.org/10.4161/derm.21923

[20] Bukhari, S.N.A., Roswandi, N.L., Waqas, M., Habib, H., Hussain, F., Khan, S., Sohail, M., Ramli, N.A., Thu, H.E. and Hussain, Z. (2018) Hyaluronic Acid, a Promising Skin Rejuvenating Biomedicine: A Review of Recent Updates and Pre-Clinical and Clinical Investigations on Cosmetic and Nutricosmetic Effects. International Journal of Biological Macromolecules, 120, 1682-1695.

https://doi.org/10.1248/bpb.19.153

[21] Rietschel, R.L. and Fowler, J.F. (1995) Fisher's Contact Dermatitis. 4th Edition, Williams \& Wilkins, Baltimore, 836.

[22] Matsuda, H., Higashino, M., Nakai, Y., Inuma, M., Kubo, M. and Lang, F.A. (1996) Studies of Cuticle Drugs from Natural Sources. IV. Inhibitory Effects of Some Arctostaphylos Plants on Melanin Biosynthesis. Biological and Pharmaceutical Bulletin, 19, 153-156. https://doi.org/10.1248/bpb.19.153

[23] West, B.J., Deng, S. and Palu, A.K. (2009) Antioxidant and Toxicity Tests of Roasted Noni (Morinda citrifolia) Leaf Infusion. International Journal of Food Science and Technology, 44, 2142-2146. https://doi.org/10.1111/j.1365-2621.2009.02051.x

[24] Everett, J.S. and Sommers, M.S. (2013) Skin Viscoelasticity: Physiologic Mechanisms, Measurement Issues, and Application to Nursing Science. Biological Research for Nursing, 15, 338-346. https://doi.org/10.1177/1099800411434151

[25] de Farias Pires T., Azambuja, A.P., Horimoto, A.R., Nakamura, M.S., de Oliveira Alvim, R., Krieger, J.E. and Pereira, A.C. (2016) A Population-Based Study of the Stratum Corneum Moisture. Clinical, Cosmetic and Investigational Dermatology, 9 , 79-87. https://doi.org/10.2147/CCID.S88485

[26] Paes, E.C., Teepen, H.J.L.J.M., Koop, W.A. and Kon, M. (2009) Perioral Wrinkles: Histologic Differences between Men and Women. Aesthetic Surgery Journal, 29, 467-472. https://doi.org/10.1016/j.asj.2009.08.018

[27] Mandfredini, M., Mazzaglia, G., Ciardo, S., Simonazzi, S., Farnetani, F., Longo, C. and Pellacani, G. (2013) Does Skin Hydration Influence Keratinocyte Biology? In Vivo Evaluation of Microscopic Skin Changes Induced by Moisturizers by Means of Reflectance Confocal Microscopy. Skin Research and Technology, 19, 299-307. https://doi.org/10.1111/srt.12042

[28] Rittie, L. and Fisher, G.J. (2015) Natural and Sun-Induced Aging of Human Skin. Cold Spring Harbor Perspectives in Medicine, 5, Article ID: a015370. https://doi.org/10.1101/cshperspect.a015370

[29] Warren, R., Gartstein, V., Kligman, A.M., Montagna, W., Allendorf, R.A. and Ridder, G.M. (1981) Age, Sunlight, and Facial Skin: A Histologic and Quantitative Study. Journal of the American Academy of Dermatology, 25, 751-670. https://doi.org/10.1016/S0190-9622(08)80964-4 
[30] El-Domyati, M., Attia, S., Saleh, F., Brown, D., Birk, D.E., Gasparro, F., Ahmad, H. and Uitto, J. (2002) Intrinsic Aging vs. Photoaging: A Comparative Histopathological, Immunohistochemical, and Ultrastructural Study of Skin. Experimental Dermatology, 11, 398-405. https://doi.org/10.1034/j.1600-0625.2002.110502.x

[31] Hanson, K.M. and Simon, J.D. (1998) Epidermal Trans-Urocanic Acid and the UV-A-Induced Photoaging of the Skin. Proceedings of the National Academy of Sciences of the United States of America, 95, 10576-10578.

[32] Brenneisen, P., Wenk, J., Klotz, L.O., Wlaschek, M., Brivina, K., Krieg, T. and Scharffetter-Kochanek, K. (1998) Central Role of Ferrous/Ferric Iron in the Ultraviolet B Irradiation-Mediated Signaling Pathway Leading to Increased Interstitial Collagenase (Matrix-Degrading Metalloprotease (MMP)-1) and Stromelysin-1 (MMP-3) mRNA Levels in Cultured Human Dermal Fibroblasts. Journal of Biological Chemistry, 273, 5279-5287. https://doi.org/10.1074/jbc.273.9.5279

[33] Rijken, F. and Bruijnzeel, P.L.B. (2009) The Pathogenesis of Photoaging: The Role of Neutrophils and Neutrophil-Derived Enzymes. Journal of Investigative Dermatology Symposium Proceedings, 14, 67-72. https://doi.org/10.1038/jidsymp.2009.15

[34] Adhikari, S., Joshi, R., Patro, B.S., Ghanty, T.K., Chintalwar, G.J., Sharma, A., Chattopadhyay, S. and Mukherjee, T. (2003) Antioxidant Activity of Bakuchiol: Experimental Evidences and Theoretical Treatments on the Possible Involvement of the Terpenoid Chain. Chemical Research in Toxicology, 16, 1062-1069. https://doi.org/10.1021/tx034082r

[35] Liu, H., Guo, W., Guo, H., Zhao, L., Yue, L., Li, X., Feng, D., Luo, J., Wu, X., Cui, W. and Qu, Y. (2020) Bakuchiol Attenuates Oxidative Stress and Neuron Damage by Regulating Trx1/TXNIP and the Phosphorylation of AMPK after Subarachnoid Hemorrhage in Mice. Frontiers in Pharmacology, 11, Article No. 712. https://doi.org/10.3389/fphar.2020.00712

[36] Emy, S., Iswari, I.S. and Winarti, N.W. (2021) Terminalia ferdinandiana Inhibited the Increase of Matrix Metalloproteinase-I and Prevent Collagen Decrease in Mice Skin Exposed to UV-B. Neurologico Spinale Medico Chirurgico, 4, 11-14. https://doi.org/10.36444/nsmc.v4i1.136

[37] Lintner, K., Gerstein, F. and Solish, N. (2020) A Serum Containing Vitamins C \& E and a Matric-Repair Tripeptide Reduces Facial Signs of Aging as Evidenced by Primos ${ }^{\circledast}$ Analysis and Frequently Repeated Auto-Perception. Journal of Cosmetic Dermatology, 19, 3262-3269. https://doi.org/10.1111/jocd.13770

[38] Sanz, M.T., Campos, C., Milani, M., Foyaca, M., Lamy, A., Kurdian, K. and Trullas, C. (2016) Biorevitalizing Effect of a Novel Facial Serum Containing Apple Stem Cell Extract, Pro-Collagen Lipopeptide, Creatine, and Urea on Skin Aging Signs. Journal of Cosmetic Dermatology, 15, 24-30. https://doi.org/10.1111/jocd.12173

[39] Herndon, J.H., Jiang, L., Kononov, T. and Fox, T. (2015) An Open Label Clinical Trial of a Multiple-Ingredient Anti-Aging Moisturizer Designed to Improve the Appearance of Facial Skin. Journal of Drugs in Dermatology, 14, 699-704. 